\title{
The cosmological backreaction: gauge (in)dependence, observers and scalars
}

\author{
G. Marozzi ${ }^{1,2}$ \\ ${ }^{1}$ Collège de France, 11 Place M. Berthelot, 75005 Paris, France \\ ${ }^{2}$ GR $\epsilon$ CO Institut d'Astrophysique de Paris, UMR7095 CNRS, \\ Université Pierre \& Marie Curie, 98 bis boulevard Arago, 75014 Paris, France
}

\begin{abstract}
We discuss several issues related to a recent proposal for defining classical spatial averages to be used in the so-called cosmological backreaction problem. In the large averaging-volume limit all gauge dependence disappears and different averages can be univocally characterized by the observers associated with different scalar fields. The relation between such averaging procedure and the standard one is emphasized and a gauge invariant way to select different observers is presented. For finite averaging volumes we show that, within our proposal, a residual gauge dependence is left, but is suppressed by several effects.
\end{abstract}




\section{Introduction}

The geometric properties of the Universe, on sufficiently large scale of distances, may be described by a homogeneous and isotropic Friedman-Lemaitre-Robertson-Walker (FLRW) metric. On the other hand on small scales the Universe exhibits a very inhomogeneous structure. In this way the FLRW metric should be interpreted as an "averaged" cosmological metric. A problem (see, for example, [1]) appears due to the fact that the Einstein equations for the averaged geometry are different, in general, from the averaged Einstein equations. In fact the averaging procedure does not commute, in general, with the nonlinear differential operators appearing in the Einstein equations. As a consequence, the dynamics of the averaged geometry is affected by so-called "backreaction" terms, originating from the dynamic contribution of the inhomogeneities present in the metric and matter sectors.

Following the discovery of cosmic acceleration on large scales, interest in these issues has considerably risen (see [2] for a review). In fact, it has been suggested (see e.g. [3]) that the dynamical effects of the backreaction could replace dark-energy sources in the explanation of such cosmic acceleration, solving, in this way, also the well-known "coincidence problem".

In order to have a physical interpretation the results that one obtains in the investigation of the backreaction problem should be independent from the gauge chosen. More generally, the gauge issue consist in extracting physically meaningful results from cosmological perturbation theory eliminating all possible gauge artifacts. At linear order in the perturbations, the way to do this is well known: following Bardeen's pioneering work [4], it consists of defining gauge invariant combinations of the perturbations themselves. The problem is more involved when one is interested in evaluating backreaction effects either at the classical or at the quantum level. Such a problem has been faced in [5], where a gauge invariant prescription to average in cosmological setup was defined, and in [6] where, using the previous prescription, a general-covariant and gauge invariant generalization of the effective equations (introduced in [7]) which describe the cosmological backreaction was given (see [8] for a specific application of the prescription introduced in [5]). The key point of our approach is to define a general formula for the classical or quantum average of any scalar quantity, on hypersurfaces on which another given scalar quantity is constant. The physical object is the hypersurface defined by the above scalar, and not the gauge chosen to calculate such an average.

The gauge issue, in connection with the backreaction, has been already partially discussed in the literature (see e.g. [9]-[11]), in particular in [11] the authors, after introducing a generalization of the effective equations presented in [7], applied the effective equations 
to two different gauges. Following $[5,6]$ the results of [11] should be seen in the following way. As already noted in [5] the standard average prescription present in the literature [7], applied in a particular gauge, can be seen as the gauge invariant prescription introduced in $[5,6]$ with the average performed on the hypersurface defined by a scalar field which is homogeneous in that particular gauge. So, from this point of view, it is clear that different gauges should lead to different results for the standard averaging procedure, since this correspond to averages performed on different hypersurfaces. The different results obtained in [11] actually correspond to the cosmological backreaction with respect to different observers/hypersurfaces. A key question comes out: which hypersurface should be chosen to perform the average? The point is to understand what are the physical observers, with respect to whom the backreaction should be calculated for the problem under consideration.

In this paper, after establishing a correspondence between the standard average prescription and the one introduced in [5,6], we will see how to define, for any given gauge, an associated homogeneous scalar. We will then show how the physical properties of the observers associated with such scalars are actually independent from the gauge chosen to perform the calculation. In particular we will present a gauge invariant description of observers in geodesic motion. Such observers have been often suggested as the physically relevant ones (see, for example, [9]) for the calculation of the cosmological backreaction of the present space-time inhomogeneities. Furthermore we will discuss in detail the residual gauge dependence of the average prescription introduced in $[5,6]$, when the average is performed on a finite volume (see Sect. 2 of [5]).

The paper is organized as follows. In Sect.2 we recall the averaging prescription introduced in $[5,6]$. In Sect.3 we give some useful relations among the scalars used to define the hypersurfaces where the average is performed. In particular the expression which defines the scalar that is homogeneous in a given gauge, and the relation which connects different homogeneous scalars in different gauges. Furthermore, we define the geodesic motion of an observer, up to second order in perturbation theory, in a perturbed FLRW space-time, and we introduce the hypersurface which defines such observers in geodesic motion independently from the gauge considered. In Sect.4 we study the residual gauge dependence of the averaging prescription as a function of the size of the volume of integration. Our conclusive remarks are presented in Sect.5.

\section{Averaging prescription: a recent proposal}

To evaluate the dynamical contribution of the inhomogeneities, present in the metric and matter sectors, one has first to introduce a well defined averaging procedure with respect to 
the observer involved in the measurements. For this purpose let us begin from the following four dimensional integral of a scalar $S(x)$ defined in [5]

$$
F(S, \Omega)=\int_{\Omega(x)} d^{4} x \sqrt{-g(x)} S(x) \equiv \int_{\mathcal{M}_{4}} d^{4} x \sqrt{-g(x)} S(x) W_{\Omega}(x) .
$$

The integration region $\Omega \subseteq \mathcal{M}_{4}$ is defined in terms of a suitable scalar window function $W_{\Omega}$, selecting a region with temporal boundaries determined by the space-like hypersurfaces $\Sigma(A)$ over which a scalar field $A(x)$ takes constant values, and with spatial boundary determined by the coordinate condition $B<r_{0}$, where $B$ is a (positive) function of the coordinates with space-like gradient $\partial_{\mu} B$, and $r_{0}$ is a positive constant. As we are interested in the variation of the volume averages along the flow lines normal to $\Sigma(A)$, we choose the following window function [6]:

$$
W_{\Omega}(x)=u^{\mu} \nabla_{\mu} \theta\left(A(x)-A_{0}\right) \theta\left(r_{0}-B(x)\right)
$$

where $\theta$ is the Heaviside step function, and $u^{\mu}=-\frac{\partial^{\mu} A}{\left(-\partial^{\tau} A \partial^{\nu} A g_{\tau \nu}\right)^{1 / 2}}$.

Using the window function (2.2) we can define the averaging of a scalar $S(x)$ as

$$
\langle S\rangle_{A_{0}, r_{0}}=\frac{F(S, \Omega)}{F(1, \Omega)}=\frac{\int d^{4} x \sqrt{-g} S u^{\mu} \nabla_{\mu} \theta\left(A(x)-A_{0}\right) \theta\left(r_{0}-B(x)\right)}{\int d^{4} x \sqrt{-g} u^{\mu} \nabla_{\mu} \theta\left(A(x)-A_{0}\right) \theta\left(r_{0}-B(x)\right)} .
$$

In order to explicitly rewrite the above definition in terms of spatial (three-dimensional) integrals we can perform the covariant derivative and exploit the properties of the consequent delta function. On considering the coordinate transformation to the bar coordinates, in which the proper time $t$ goes to $\bar{t}$ defined by $t=h(\bar{t}, x)$ and where the function $h$ is chosen to make the scalar field $A$ homogeneous, i.e.

$$
A(h(\bar{t}, x), x)=\bar{A}(\bar{t}, x) \equiv A^{(0)}(\bar{t})
$$

one can perform the time integration and obtain

$$
\langle S\rangle_{A_{0}, r_{0}}=\frac{\int_{\Sigma_{A_{0}}} d^{3} x \sqrt{\left|\bar{\gamma}\left(t_{0}, \vec{x}\right)\right|} \bar{S}\left(t_{0}, \vec{x}\right) \theta\left(r_{0}-B\left(h\left(t_{0}, \vec{x}\right), \vec{x}\right)\right.}{\int_{\Sigma_{A_{0}}} d^{3} x \sqrt{\left|\bar{\gamma}\left(t_{0}, \vec{x}\right)\right|} \theta\left(r_{0}-B\left(h\left(t_{0}, \vec{x}\right), \vec{x}\right)\right)}
$$

where we have called $t_{0}$ the time $\bar{t}$ when $A^{(0)}(\bar{t})$ takes the constant values $A_{0}$ and we are averaging on a section of the three-dimensional hypersurface $\Sigma_{A_{0}}$, hypersurface where $A(x)=A_{0}$. In this notation, for example, $\bar{S}$ is the variable $S$ transformed to the coordinate frame in which $A(x)$ is homogeneous. In this framework the hypersurface $A(x)=A_{0}$ is the one which identifies our observers (see Sect. 3).

As discussed in [5], if $B$ is not a scalar, the spatial boundary can be a source of breaking of covariance and gauge invariance. In the limit of large averaging volume with respect 
to the typical scale of the inhomogeneities, all the gauge dependence disappears ([5]) and the classical average, or the correspondent vacuum expectation value (see [5]), is uniquely characterized by the hypersurface of integration $A(x)=A_{0}$ regardless from the gauge chosen to perform the calculation. In Sect. 3 we will give some useful tool to define the scalar $A(x)$ and, using a particular example, we will see as the physical properties of the observers associated to such scalar are independent from the gauge chosen, while in Sect.4 we will discuss in more quantitative terms such a breaking.

\section{Gauges vs observers in a gauge invariant averaging pre- scription}

As stated in $[5,6]$ and in the introduction, backreaction effects are usually calculated, in the literature, in different gauges using the standard averaging prescription [7]. In this way the results can be seen as particular cases of the average prescription recalled in Sect.2 only if the gauge considered is the one where the scalar $A(x)$, which defines the hypersurface on which we perform the average, is homogeneous. In such a gauge, using the ADM formalism, the standard averaging prescription [7] is also covariantly defined. In [7] this corresponds to identify $A(x)$ with the scalar potential of the fluid and choose the so-called covariant fluid gauge [12].

To better understand the connection above let us see in detail how it is always possible to define a particular scalar which is homogeneous in a particular given gauge.

\subsection{Scalars/Gauges correspondence}

We shall work in the context of a spatially flat, FLRW background geometry, and we expand our background fields $\left\{g_{\mu \nu}\right\}$ up to second order in the non-homogeneous perturbations, without fixing any gauge, as follows:

$$
\begin{aligned}
g_{00}= & -1-2 \alpha^{(1)}-2 \alpha^{(2)}, \quad g_{i 0}=-\frac{a}{2}\left(\beta_{, i}^{(1)}+B_{i}^{(1)}\right)-\frac{a}{2}\left(\beta_{, i}^{(2)}+B_{i}^{(2)}\right), \\
g_{i j}= & a^{2}\left[\delta_{i j}\left(1-2 \psi^{(1)}-2 \psi^{(2)}\right)+D_{i j}\left(E^{(1)}+E^{(2)}\right)+\frac{1}{2}\left(\chi_{i, j}^{(1)}+\chi_{j, i}^{(1)}+h_{i j}^{(1)}\right)\right. \\
& \left.+\frac{1}{2}\left(\chi_{i, j}^{(2)}+\chi_{j, i}^{(2)}+h_{i j}^{(2)}\right)\right],
\end{aligned}
$$

where $D_{i j}=\partial_{i} \partial_{j}-\delta_{i j}\left(\nabla^{2} / 3\right)$, and $a=a(t)$ is the scale factor of the homogeneous FLRW metric. Here $\alpha^{(1)}, \beta^{(1)}, \psi^{(1)}, E^{(1)}$ are pure scalar first-order perturbations, $B_{i}^{(1)}$ and $\chi_{i}^{(1)}$ are transverse vectors $\left(\partial^{i} B_{i}^{(1)}=0\right.$ and $\left.\partial^{i} \chi_{i}^{(1)}=0\right), h_{i j}^{(1)}$ is a traceless and transverse ten- 
sor $\left(\partial^{i} h_{i j}^{(1)}=0=h_{i}^{(1) i}\right)$, and the same notation applies to the case of the second-order perturbations.

From Eq.(3.1) one obtains 10 degrees of freedom (which must be added to those coming from the matter sector) which are in part redundant. To obtain a set of well defined equations (Einstein equations + equations of motion of the matter sector), order by order, we have, for example, to set to zero two scalar perturbations and one vector perturbation.

The choice of such variables is called a "gauge" choice and can be performed using the gauge transformation, associated to a "infinitesimal" coordinate transformation, from the general gauge (3.1) to the particular gauge chosen. Such "infinitesimal" coordinate transformation is parametrized by the first-order, $\epsilon_{(1)}^{\mu}$, and second-order, $\epsilon_{(2)}^{\mu}$, generators as $[13]$

$$
x^{\mu} \rightarrow \tilde{x}^{\mu}=x^{\mu}+\epsilon_{(1)}^{\mu}+\frac{1}{2}\left(\epsilon_{(1)}^{\nu} \partial_{\nu} \epsilon_{(1)}^{\mu}+\epsilon_{(2)}^{\mu}\right)+\ldots
$$

where

$$
\epsilon_{(1)}^{\mu}=\left(\epsilon_{(1)}^{0}, \hat{\epsilon}_{(1)}^{i}\right), \quad \epsilon_{(2)}^{\mu}=\left(\epsilon_{(2)}^{0}, \hat{\epsilon}_{(2)}^{i}\right)
$$

and we can explicitly separate the scalar and the pure transverse vector part of $\hat{\epsilon}_{(1)}^{i}, \hat{\epsilon}_{(2)}^{i}$ as

$$
\hat{\epsilon}_{(1)}^{i}=\partial^{i} \epsilon_{(1)}+\epsilon_{(1)}^{i} \quad, \quad \hat{\epsilon}_{(2)}^{i}=\partial^{i} \epsilon_{(2)}+\epsilon_{(2)}^{i} .
$$

Under the associated gauge transformation (or local field reparametrization) - where, by definition, old and new fields are evaluated at the same space-time point $\mathrm{x}$ - a general tensor changes, to first and second order, as

$$
\begin{gathered}
T^{(1)} \rightarrow \tilde{T}^{(1)}=T^{(1)}-L_{\epsilon_{(1)}} T^{(0)} \\
T^{(2)} \rightarrow \tilde{T}^{(2)}=T^{(2)}-L_{\epsilon_{(1)}} T^{(1)}+\frac{1}{2}\left(L_{\epsilon_{(1)}}^{2} T^{(0)}-L_{\epsilon_{(2)}} T^{(0)}\right)
\end{gathered}
$$

where $L_{\epsilon_{(1,2)}}$ is the Lie derivative respect the vector $\epsilon_{(1,2)}^{\mu}$. In particular the scalar metric perturbations change, to first order, as

$$
\begin{gathered}
\tilde{\alpha}^{(1)}=\alpha^{(1)}-\dot{\epsilon}_{(1)}^{0}, \\
\tilde{\beta}^{(1)}=\beta^{(1)}-\frac{2}{a} \epsilon_{(1)}^{0}+2 a \dot{\epsilon}_{(1)}, \\
\tilde{\psi}^{(1)}=\psi^{(1)}+H \epsilon_{(1)}^{0}+\frac{1}{3} \nabla^{2} \epsilon_{(1)}, \\
\tilde{E}^{(1)}=E^{(1)}-2 \epsilon_{(1)} .
\end{gathered}
$$


and to second order, as

$$
\begin{aligned}
\tilde{\alpha}^{(2)}= & \alpha^{(2)}-\frac{1}{2} \dot{\epsilon}_{(2)}^{0}-\epsilon_{(1)}^{0} \dot{\alpha}^{(1)}-2 \alpha^{(1)} \dot{\epsilon}_{(1)}^{0}+\frac{1}{2} \epsilon_{(1)}^{0} \ddot{\epsilon}_{(1)}^{0}+\left(\dot{\epsilon}_{(1)}^{0}\right)^{2}-\hat{\epsilon}_{(1)}^{i} \alpha_{, i}^{(1)} \\
& +\frac{1}{2}\left(\hat{\epsilon}_{(1)}^{i} \epsilon_{(1), i}^{0}\right) \cdot-\frac{a^{2}}{2} \dot{\hat{\epsilon}}_{(1)}^{i} \dot{\hat{\epsilon}}_{(1) i}-\frac{a}{2}\left(\beta_{, i}^{(1)}+B_{i}^{(1)}\right) \dot{\hat{\epsilon}}_{(1)}^{i}, \\
\tilde{\beta}^{(2)=} & \beta^{(2)}-\frac{1}{a} \epsilon_{(2)}^{0}+a \dot{\epsilon}_{(2)}+\frac{1}{2 a} \frac{d}{d t}\left(\epsilon_{(1)}^{0}\right)^{2}+\frac{1}{a} \hat{\epsilon}_{(1)}^{i} \epsilon_{(1), i}^{0}-\hat{\epsilon}_{(1)}^{i} \beta_{, i}^{(1)}+\frac{\partial^{i}}{\nabla^{2}} \Upsilon_{i}, \\
\tilde{\psi}^{(2)}= & \psi^{(2)}+\frac{H}{2} \epsilon_{(2)}^{0}+\frac{1}{6} \nabla^{2} \epsilon_{(2)}-\epsilon_{(1)}^{0}\left(2 H \psi^{(1)}+\dot{\psi}^{(1)}\right)-\frac{H}{2} \epsilon_{(1)}^{0} \dot{\epsilon}_{(1)}^{0} \\
& -\frac{\epsilon_{(1)}^{02}}{2}\left(\dot{H}+2 H^{2}\right)-\frac{H}{2} \epsilon_{(1), i}^{0} \hat{\epsilon}_{(1)}^{i}-\psi_{, i}^{(1)} \hat{\epsilon}_{(1)}^{i}-\frac{1}{6} \Pi_{i}^{i}, \\
\tilde{E}^{(2)}= & E^{(2)}-\epsilon_{(2)}-\frac{1}{2} \frac{1}{\nabla^{2}} \Pi_{i}^{i}+\frac{3}{2} \frac{\partial^{i} \partial^{j}}{\left(\nabla^{2}\right)^{2}} \Pi_{i j},
\end{aligned}
$$

where

$$
\begin{aligned}
\Upsilon_{i}= & \frac{2}{a} \epsilon_{(1), i}^{0} \dot{\epsilon}_{(1)}^{0}-\epsilon_{(1)}^{0}\left(\dot{\beta}_{, i}^{(1)}+\dot{B}_{i}^{(1)}\right)-H \epsilon_{(1)}^{0}\left(\beta_{, i}^{(1)}+B_{i}^{(1)}\right)-\dot{\epsilon}_{(1)}^{0}\left(\beta_{, i}^{(1)}+B_{i}^{(1)}\right) \\
& \quad-\frac{4}{a} \alpha^{(1)} \epsilon_{(1), i}^{0}-2 a \dot{\hat{\epsilon}}_{(1) i}\left(2 H \epsilon_{(1)}^{0}+\frac{1}{2} \dot{\epsilon}_{(1)}^{0}+2 \psi^{(1)}\right)-a \ddot{\hat{\epsilon}}_{(1) i} \epsilon_{(1)}^{0} \\
& -a\left(\hat{\epsilon}_{(1)}^{j} \dot{\hat{\epsilon}}_{(1) i, j}+2 \hat{\epsilon}_{(1), i}^{j} \dot{\hat{\epsilon}}_{(1) j}+\dot{\hat{\epsilon}}_{(1)}^{j} \hat{\epsilon}_{(1) i, j}\right)-\hat{\epsilon}_{(1)}^{j} B_{i, j}^{(1)}-\hat{\epsilon}_{(1), i}^{j} B_{j}^{(1)} \\
& -2 a \dot{\hat{\epsilon}}_{(1)}^{j}\left[-D_{i j} E^{(1)}-\frac{1}{2}\left(\chi_{i, j}^{(1)}+\chi_{j, i}^{(1)}-h_{i j}^{(1)}\right)\right] \\
\Pi_{i j}=+ & 2 \epsilon_{(1)}^{0} D_{i j}\left(H E^{(1)}+\frac{\dot{E}^{(1)}}{2}\right)-\hat{\epsilon}_{(1)}^{k} D_{i j} E_{, k}^{(1)}-\hat{\epsilon}_{(1), j}^{k} D_{i k} E^{(1)}-\hat{\epsilon}_{(1), i}^{k} D_{j k} E^{(1)} \\
+ & 2 H \epsilon_{(1)}^{0}\left(\hat{\epsilon}_{(1) i, j}+\hat{\epsilon}_{(1) j, i}\right)+2 \psi^{(1)}\left(\hat{\epsilon}_{(1) i, j}+\hat{\epsilon}_{(1) j, i}\right)-\epsilon_{(1)}^{0}\left[H\left(\chi_{i, j}^{(1)}+\chi_{j, i}^{(1)}+h_{i j}^{(1)}\right)\right. \\
+ & \left.\frac{1}{2}\left(\dot{\chi}_{i, j}^{(1)}+\dot{\chi}_{j, i}^{(1)}+\dot{h}_{i j}^{(1)}\right)\right]+\frac{1}{2} \epsilon_{(1)}^{0}\left(\dot{\hat{\epsilon}}_{(1) i, j}+\dot{\hat{\epsilon}}_{(1) j, i}\right)-\frac{1}{a^{2}} \epsilon_{(1), i}^{0} \epsilon_{(1), j}^{0} \\
+ & \hat{\epsilon}_{(1) k, i} \hat{\epsilon}_{(1), j}^{k}+\frac{1}{2} \hat{\epsilon}_{(1)}^{k}\left(\hat{\epsilon}_{(1) i, j k}+\hat{\epsilon}_{(1) j, i k}\right)-\frac{1}{2} \hat{\epsilon}_{(1)}^{k}\left(\chi_{i, j k}^{(1)}+\chi_{j, i k}^{(1)}+h_{i j, k}^{(1)}\right) \\
+ & \frac{1}{2}\left(\dot{\hat{\epsilon}}_{(1) i} \epsilon_{(1), j}^{0}+\dot{\hat{\epsilon}}_{(1) j} \epsilon_{(1), i}^{0}\right)+\frac{1}{2 a}\left(\beta_{, i}^{(1)}+B_{i}^{(1)}\right) \epsilon_{(1), j}^{0}+\frac{1}{2 a}\left(\beta_{, j}^{(1)}+B_{j}^{(1)}\right) \epsilon_{(1), i}^{0} \\
+ & \frac{1}{2}\left(\hat{\epsilon}_{(1) j, k} \hat{\epsilon}_{(1), i}^{k}+\hat{\epsilon}_{(1) i, k} \hat{\epsilon}_{(1), j}^{k}\right)-\frac{1}{2}\left(\chi_{i, k}^{(1)}+\chi_{k, i}^{(1)}+h_{i k}^{(1)}\right) \hat{\epsilon}_{(1), j}^{k} \\
+ & \frac{1}{2}\left(\chi_{j, k}^{(1)}+\chi_{k, j}^{(1)}+h_{j k}^{(1)}\right) \hat{\epsilon}_{(1), i}^{k} .
\end{aligned}
$$

For the vector metric perturbations one obtains, to first order

$$
\begin{gathered}
\tilde{B}_{i}^{(1)}=B_{i}^{(1)}+2 a \dot{\epsilon}_{(1) i} \\
\tilde{\chi}_{i}^{(1)}=\chi_{i}^{(1)}-2 \epsilon_{(1) i}
\end{gathered}
$$


and to second order [14]

$$
\begin{gathered}
\tilde{B}_{i}^{(2)}=B_{i}^{(2)}+a \dot{\epsilon}_{(2) i}-\frac{\partial_{i} \partial^{j}}{\nabla^{2}} \Upsilon_{j}+\Upsilon_{i} \\
\tilde{\chi}_{i}^{(2)}=\chi_{i}^{(2)}-\epsilon_{(2) i}-2 \frac{\partial_{i} \partial^{j} \partial^{k}}{\left(\nabla^{2}\right)^{2}} \Pi_{j k}+2 \frac{\partial^{j}}{\nabla^{2}} \Pi_{i j}
\end{gathered}
$$

The metric tensor perturbation is gauge invariant to first order $\left(\tilde{h}_{i j}^{(1)}=h_{i j}^{(1)}\right)$ while to second order one obtains [14]

$$
\tilde{h}_{i j}^{(2)}=h_{i j}^{(2)}+2 \Pi_{i j}+\left(\frac{\partial_{i} \partial_{j}}{\nabla^{2}}-\delta_{i j}\right) \Pi_{k}^{k}+\left(\frac{\partial_{i} \partial_{j}}{\nabla^{2}}+\delta_{i j}\right) \frac{\partial^{k} \partial^{l}}{\nabla^{2}} \Pi_{k l}-\frac{2}{\nabla^{2}}\left(\partial_{i} \partial^{k} \Pi_{j k}+\partial_{j} \partial^{k} \Pi_{i k}\right) .
$$

Let us note that the differences between Eqs.(3.9,3.10)-(3.13,3.14) and corresponding ones of [14] are connected with a slightly different definition of the scalar perturbations associated with $g_{i j}$.

To conclude the associated gauge transformation of a scalar field $A(x)=A^{(0)}(t)+$ $A^{(1)}(x)+A^{(2)}(x)$ is, to first order,

$$
A^{(1)} \rightarrow \widetilde{A}^{(1)}=A^{(1)}-\epsilon_{(1)}^{0} \dot{A}^{(0)}
$$

and, to second order,

$$
\begin{aligned}
A^{(2)} \rightarrow \quad & \widetilde{A}^{(2)}=A^{(2)}-\epsilon_{(1)}^{0} \dot{A}^{(1)}-\left(\epsilon_{(1)}^{i}+\partial^{i} \epsilon_{(1)}\right) \partial_{i} A^{(1)} \\
+ & \frac{1}{2}\left[\epsilon_{(1)}^{0} \partial_{t}\left(\epsilon_{(1)}^{0} \dot{A}^{(0)}\right)+\left(\epsilon_{(1)}^{i}+\partial^{i} \epsilon_{(1)}\right) \partial_{i} \epsilon_{(1)}^{0} \dot{A}^{(0)}-\epsilon_{(2)}^{0} \dot{A}^{(0)}\right] .
\end{aligned}
$$

In this way the general scalar $A(x)$ homogeneus in the gauge chosen (identified by the generators $\epsilon_{(1)}^{\mu}$ and $\left.\epsilon_{(2)}^{\mu}\right)$ will be given by

$$
\begin{aligned}
& A^{(0)}(t)=A^{(0)}(t) \\
& A^{(1)}(x)=\dot{A}^{(0)} \epsilon_{(1)}^{0} \\
& A^{(2)}(x)=\frac{\dot{A}^{(0)}}{2} \epsilon_{(1)}^{0} \dot{\epsilon}_{(1)}^{0}+\frac{\ddot{A}^{(0)}}{2} \epsilon_{(1)}^{02}+\frac{\dot{A}^{(0)}}{2}\left[\left(\epsilon_{(1)}^{i}+\partial^{i} \epsilon_{(1)}\right) \partial_{i} \epsilon_{(1)}^{0}+\epsilon_{(2)}^{0}\right]
\end{aligned}
$$

we have a class of scalars defined by their homogeneous value $A^{(0)}(t)$ which can take any value. From another point of view, the coordinate transformation, that goes from a general gauge to the bar gauge where $A(x)$ is homogeneous, fixes the corresponding transformation parameters as follows:

$$
\epsilon_{(1)}^{0}=\frac{A^{(1)}}{\dot{A}^{(0)}}, \quad \epsilon_{(2)}^{0}=2 \frac{A^{(2)}}{\dot{A}^{(0)}}-\frac{A^{(1)} \dot{A^{(1)}}}{\left(\dot{A}^{(0)}\right)^{2}}-\frac{1}{\dot{A}^{(0)}}\left(\partial^{i} \epsilon_{(1)}+\epsilon_{(1)}^{i}\right) \partial_{i} A^{(1)}
$$


as it is easy to control the two set of equations (3.24) and (3.25) are one the inverse of the other.

To check the results in Eqs. (3.24) one can consider the simple case of a space-time sourced by a single scalar field $\phi(x)=\phi^{(0)}(t)+\varphi^{(1)}(x)+\varphi^{(2)}(x)$, and choose the uniform field gauge (UFG), namely the gauge for which such scalar $\phi(x)$ is homogeneous, as the gauge where our scalar $A(x)$ should be homogeneous. In such a case is pretty obvious that $A(x)$ is given by a general function of $\phi(x)$, namely $A=A(\phi(x))$. If we now expand up to second order in perturbation theory we obtain

$$
A(x)=A(\phi(x))=A^{(0)}+\frac{\dot{A}^{(0)}}{\dot{\phi}^{(0)}} \varphi^{(1)}+\frac{\dot{A}^{(0)}}{\dot{\phi}^{(0)}} \varphi^{(2)}+\frac{\dot{A}^{(0)}}{2 \dot{\phi}^{(0)} 2}\left(\frac{\ddot{A}^{(0)}}{\dot{A}^{(0)}}-\frac{\ddot{\phi}^{(0)}}{\dot{\phi}^{(0)}}\right) \varphi^{(1) 2},
$$

which is, in fact, the expected solution of Eqs. (3.24).

As pointed out the scalar $A(x)$ is used to identify our observers. They are the ones sitting on the spacelike hypersurfaces over which the scalar field $A(x)$ takes constant values, and their motion is given by the comoving 4-velocity

$$
u_{\mu}=-\frac{\partial_{\mu} A}{\left(-\partial_{\sigma} A \partial^{\sigma} A\right)^{1 / 2}}
$$

which takes the general form

$$
\begin{aligned}
u_{\mu}^{(0)}= & (-1, \overrightarrow{0}) \\
u_{\mu}^{(1)}= & \left(-\alpha^{(1)},-\epsilon_{(1), i}^{0}\right) \\
u_{\mu}^{(2)}= & \left(\frac{\alpha^{(1) 2}}{2}+\frac{\epsilon_{(1), j}^{0}}{2 a}\left(\beta^{(1), j}+B^{(1) j}\right)-\frac{1}{2 a^{2}} \epsilon_{(1), j}^{0} \epsilon_{(1)}^{0, j}-\frac{1}{8}\left(\beta_{, j}^{(1)}+B_{j}^{(1)}\right)\left(\beta^{(1), j}+B^{(1) j}\right)-\alpha^{(2)},\right. \\
& \left.\frac{1}{2} \epsilon_{(1), i}^{0} \dot{\epsilon}_{(1)}^{0}-\frac{1}{2} \epsilon_{(1)}^{0} \dot{\epsilon}_{(1), i}^{0}-\alpha^{(1)} \epsilon_{(1), i}^{0}-\frac{\epsilon_{(2), i}^{0}}{2}-\frac{1}{2}\left(\hat{\epsilon}_{(1), i}^{j} \epsilon_{(1), j}^{0}+\hat{\epsilon}_{(1)}^{j} \epsilon_{(1), j i}^{0}\right)\right)
\end{aligned}
$$

This normal vector to the hypersurface is the one which describes the physical properties of our observers. As we will see in a particular example in the next subsection, such properties are the same in all possible gauges, the vector describes a gauge invariant set of properties. Let us also put in evidence that such a vector is independent from the homogeneous value $A^{(0)}(t)$ of the corresponding scalar. In general, this value can be chosen at will and does not influence the physical properties of the observers and the physical meaning of our averages. For example, we can choose $A^{(0)}(t)=t$ to have standard derivatives in the gauge invariant formulation of the cosmological backreaction [6].

An interesting result is that all these particular scalars, homogeneous in different gauges, can be connected to each other by a simple formula. Let us call with $A_{i}$ and $A_{j}$ the general 
expressions, without fixing any gauge, of the scalars homogeneous in the gauge $i$ and $j$, one obtains the following result

$$
A_{i}=A_{j}-\left(A_{j}^{(1)}\right)_{i}-\left(A_{j}^{(2)}\right)_{i}-\left(\epsilon_{(1)}^{\mu}\right)_{i} \partial_{\mu}\left(A_{j}^{(1)}\right)_{i}
$$

where $\left(A_{j}^{(n)}\right)_{i}$ is the value that $A_{j}^{(n)}$ takes after a gauge transformation from the general gauge to the gauge $i$ (so it is gauge invariant for construction), and $\left(\epsilon_{(1)}^{\mu}\right)_{i}$ is the value that we have to give to the parameter $\epsilon_{(1)}^{\mu}$ to go from a general gauge to the particular gauge $i$. It is easy to understand that this last quantity changes in the following way under a gauge transformation identified by a generator $\epsilon_{(1)}^{\mu}$

$$
\left(\epsilon_{(1)}^{\mu}\right)_{i} \rightarrow\left(\epsilon_{(1)}^{\mu}\right)_{i}-\epsilon_{(1)}^{\mu}
$$

so the quantity obtained by using Eq.(3.29) is homogeneous in the gauge $i$ and changes as a scalar up to second order. Starting with a scalar homogeneous in a given gauge we can easily obtain a scalar homogeneous in any other given gauge using Eq.(3.29). This is a general formula that can be generalized to any order.

To conclude we have that any given gauge has a class of scalar fields, homogeneous in that gauge, while for any given scalar there is a class of bar gauges (the parameters of the coordinate transformation which gives the bar gauge are only partially determined by $A(x)$, see Eq.(3.25)).

\subsection{A privileged observer?}

One of the central problems in the calculation of backreaction effects from inhomogeneities is to choose the hypersurface with respect to which our observers are defined. Such a choice will obviously depend on the particular backreaction problem considered. In the particular case of the backreaction of large scale structure on the present cosmological evolution different authors (see, for example, [9]) have claimed that such physical observers are given by the observers in geodesic motion. This property should be a gauge independent property of our observers, i.e. the results of the backreaction should depend only on the hypersurface which defines the observers and not on the gauge chosen to perform the calculation.

An observer in geodesic motion is described by a velocity vector $v_{\mu}$ (normalized as $v_{\mu} v^{\mu}=-1$ ) which satisfies the following equation

$$
g^{\sigma \tau} v_{\sigma} \nabla_{\tau} v_{\mu}=0
$$

using the perturbed FLRW metric given in Eq.(3.1) we obtain the following result up to second order in perturbation theory

$$
v_{\mu}^{(0)}=(-1, \overrightarrow{0})
$$




$$
\begin{aligned}
& v_{\mu}^{(1)}=\left(-\alpha^{(1)},-\int d t \alpha_{, i}^{(1)}\right) \\
& v_{\mu}^{(2)}=\left(v_{0}^{(2)}, \int d t v_{0, i}^{(2)}\right)
\end{aligned}
$$

where

$$
\begin{aligned}
v_{0}^{(2)}= & \frac{\alpha^{(1) 2}}{2}-\frac{1}{2 a^{2}}\left(\int d t \alpha_{, j}^{(1)}\right)\left(\int d t \alpha^{(1), j}\right)+\frac{1}{2 a}\left(\int d t \alpha_{, j}^{(1)}\right)\left(\beta^{(1), j}+B^{(1) j}\right) \\
& -\frac{1}{8}\left(\beta_{, j}^{(1)}+B_{j}^{(1)}\right)\left(\beta^{(1), j}+B^{(1) j}\right)-\alpha^{(2)}
\end{aligned}
$$

Let us note that this vector $v_{\mu}$ will take the standard zero order form $(-1, \overrightarrow{0})$ in the socalled synchronous gauge (where $\alpha^{(1)}=\alpha^{(2)}=0, \beta^{(1)}=0$ and $B_{i}^{(1)}=0$ ). This suggests that the scalar which defines the "right" hypersurface should be the one which is homogeneous in such a synchronous gauge. Such scalar field $A(x)$ can be constructed starting from Eqs.(3.24) or using the relation in (3.29) and the scalar homogeneus in the UFG given in (3.26). After some calculation we obtain that this is given by

$$
\begin{aligned}
& A^{(0)}(t)=A^{(0)}(t) \\
& A^{(1)}(x)=\dot{A}^{(0)} \int d t \alpha^{(1)} \\
& A^{(2)}(x)=\frac{1}{2} \ddot{A}^{(0)}\left(\int d t \alpha^{(1)}\right)^{2}+\dot{A}^{(0)} \int d t\left[-\frac{1}{2} \alpha^{(1) 2}+\frac{1}{2 a^{2}}\left(\int d t \alpha_{, j}^{(1)}\right)\left(\int d t \alpha^{(1), j}\right)\right. \\
&\left.-\frac{1}{2 a}\left(\int d t \alpha_{, j}^{(1)}\right)\left(\beta^{(1), j}+B^{(1) j}\right)+\frac{1}{8}\left(\beta_{, j}^{(1)}+B_{j}^{(1)}\right)\left(\beta^{(1), j}+B^{(1) j}\right)+\alpha^{(2)}\right]
\end{aligned}
$$

and we can easily check that

$$
u_{\mu} \equiv v_{\mu},
$$

namely the observers defined by this scalar are always in geodesic motion independently from the gauge chosen. This is a gauge independent property namely a physical property of the observers.

\section{Gauge dependence for finite averaging volume}

In the calculation of the backreaction effects the region of integration has a typical size that goes from tens of Mpc to the Hubble radius. Consequently, it is important to investigate how the average introduced in $[5,6]$ depends on the gauge chosen as a consequence of the finiteness of the region of integration. The gauge dependence, up to second order in the cosmological perturbations, of a 4-dimensional integral, using the window function (2.2) and the coordinate trasformation (3.2), is given by (see, also, [5])

$$
\widetilde{F}(\widetilde{S}, \Omega)-F(S, \Omega)=-\int d^{4} x \sqrt{-g} S(x) u^{\mu} \nabla_{\mu} \theta\left(A(x)-A_{0}\right) \delta\left(r_{0}-B(x)\right)
$$




$$
\left\{\frac{\partial B(x)}{\partial x^{\mu}} \epsilon_{(1)}^{\mu}+\frac{1}{2} \frac{\partial B(x)}{\partial x^{\mu}}\left[\epsilon_{(1)}^{\nu} \partial_{\nu} \epsilon_{(1)}^{\mu}+\epsilon_{(2)}^{\mu}\right]+\frac{1}{2} \frac{\partial^{2} B(x)}{\partial x^{\mu} \partial x^{\nu}} \epsilon_{(1)}^{\mu} \epsilon_{(1)}^{\nu}\right\}
$$

where $\mathrm{a}^{\sim}$ indicates gauge trasformed quantities. Using this result in the averaging prescription defined in (2.3) one obtains:

$$
\begin{aligned}
&\langle\tilde{S}\rangle_{A_{0}, r_{0}}=\frac{\tilde{F}(\tilde{S}, \Omega)}{\tilde{F}(1, \Omega)}=\left\{F(S, \Omega)-\int d^{4} x \sqrt{-g} S(x) u^{\mu} \nabla_{\mu} \theta\left(A(x)-A_{0}\right) \delta\left(r_{0}-B(x)\right)\right. \\
& {\left.\left[\frac{\partial B(x)}{\partial x^{\mu}} \epsilon_{(1)}^{\mu}+\frac{1}{2} \frac{\partial B(x)}{\partial x^{\mu}}\left[\epsilon_{(1)}^{\nu} \partial_{\nu} \epsilon_{(1)}^{\mu}+\epsilon_{(2)}^{\mu}\right]+\frac{1}{2} \frac{\partial^{2} B(x)}{\partial x^{\mu} \partial x^{\nu}} \epsilon_{(1)}^{\mu} \epsilon_{(1)}^{\nu}\right]\right\} } \\
&\left\{F(1, \Omega)-\int d^{4} x \sqrt{-g} u^{\mu} \nabla_{\mu} \theta\left(A(x)-A_{0}\right) \delta\left(r_{0}-B(x)\right)\right. \\
& {\left.\left[\frac{\partial B(x)}{\partial x^{\mu}} \epsilon_{(1)}^{\mu}+\frac{1}{2} \frac{\partial B(x)}{\partial x^{\mu}}\left[\epsilon_{(1)}^{\nu} \partial_{\nu} \epsilon_{(1)}^{\mu}+\epsilon_{(2)}^{\mu}\right]+\frac{1}{2} \frac{\partial^{2} B(x)}{\partial x^{\mu} \partial x^{\nu}} \epsilon_{(1)}^{\mu} \epsilon_{(1)}^{\nu}\right]\right\}^{-1} }
\end{aligned}
$$

Keeping only terms up to second order in the cosmological perturbations, one obtains, after some algebra, the following result for the gauge dependence:

$$
\begin{aligned}
\langle\tilde{S}\rangle_{A_{0}, r_{0}}-\langle S\rangle_{A_{0}, r_{0}}= & \frac{\int_{\Sigma_{A_{0}}} d^{3} x \bar{S}^{(1)} \theta\left(r_{0}-B\left(t_{0}, \vec{x}\right)\right)}{\int_{\Sigma_{A_{0}}} d^{3} x \theta\left(r_{0}-B\left(t_{0}, \vec{x}\right)\right)} \frac{\int_{\Sigma_{A_{0}}} d^{3} x \frac{\partial B}{\partial x^{\mu}} \bar{\epsilon}_{(1)}^{\mu} \delta\left(r_{0}-B\left(t_{0}, \vec{x}\right)\right)}{\int_{\Sigma_{A_{0}}} d^{3} x \theta\left(r_{0}-B\left(t_{0}, \vec{x}\right)\right)} \\
& -\frac{\int_{\Sigma_{A_{0}}} d^{3} x \bar{S}^{(1)} \frac{\partial B}{\partial x^{\mu}} \bar{\epsilon}_{(1)}^{\mu} \delta\left(r_{0}-B\left(t_{0}, \vec{x}\right)\right)}{\int_{\Sigma_{A_{0}}} d^{3} x \theta\left(r_{0}-B\left(t_{0}, \vec{x}\right)\right)}
\end{aligned}
$$

where $\bar{\epsilon}_{(1)}^{\mu}$ is the first order vector generator of the gauge transformation of a bar quantity. Namely, if we consider a scalar and vector quantity in the bar coordinate and we apply a gauge transformation we obtain new quantities that can be obtained by the general definition of a gauge transformation with $\epsilon_{(1)}^{\mu}$ substituted by $\bar{\epsilon}_{(1)}^{\mu}$. In practice we obtain that

$$
\bar{\epsilon}_{(1)}^{\mu}=\left(0, \hat{\epsilon}_{(1)}^{i}\right) .
$$

Let us remark some points about this result: first, the result is independent from the window function used to carry out the classical average. If we repeat the procedure starting from $W_{\Omega}(x)=\delta\left(A(x)-A_{0}\right) \theta\left(r_{0}-B(x)\right)$ instead of $u^{\mu} \nabla_{\mu} \theta\left(A(x)-A_{0}\right) \theta\left(r_{0}-B(x)\right)$ we obtain the same result. In general, we obtain the result in Eq.(4.3) starting from a window function $\delta\left(A(x)-A_{0}\right) \theta\left(r_{0}-B(x)\right) C(x)$ with $C(x)$ any scalar field with a non-vanishing homogeneous value. Second, the gauge dependence goes to zero for $r_{0} \rightarrow+\infty$. Third, regardless of the scalar $S$ the gauge dependence is always at least of second order, so this will be always subleading if in the average $\langle S\rangle_{A_{0}, r_{0}}$ there is a non zero first order contribution. And, in the end, if $\bar{S}^{(1)}=0$ the average is gauge invariant up to second order. 


\subsection{An example}

Let us now consider a simple example where we take a sphere as spatial boundary. Namely we take $B\left(t_{0}, \vec{x}\right)=\left(x^{2}+y^{2}+z^{2}\right)^{1 / 2}=|\vec{x}|$. Having set the spatial boundary our result will depend only on the structure of $\bar{S}^{(1)}$ and $\hat{\epsilon}_{(1)}^{i}$. In general, if we consider a cosmological background sourced by an energy-momentum tensor with no transverse part for $T_{i}^{0}$, namely with no term $\hat{T}_{i}^{0}$ such that $\partial^{i} \hat{T}_{i}^{0}=0$, we have that the first order vector perturbations can be set to zero [15] regardless of the gauge and we can set, from Eq.(4.4), $\bar{\epsilon}_{(1)}^{\mu}=\left(0, \partial^{i} \epsilon_{(1)}\right)$.

Using these assumptions Eq.(4.3) can be simplified to

$$
\begin{aligned}
\langle\tilde{S}\rangle_{A_{0}, r_{0}}-\langle S\rangle_{A_{0}, r_{0}}= & \frac{\int d^{3} x \bar{S}^{(1)} \theta\left(r_{0}-|\vec{x}|\right)}{\frac{4}{3} \pi r_{0}^{3}} \frac{\int d^{3} x \vec{\nabla}|\vec{x}| \cdot \vec{\nabla} \epsilon_{(1)} \delta\left(r_{0}-|\vec{x}|\right)}{\frac{4}{3} \pi r_{0}^{3}} \\
& -\frac{\int d^{3} x \bar{S}^{(1)} \vec{\nabla}|\vec{x}| \cdot \vec{\nabla} \epsilon_{(1)} \delta\left(r_{0}-|\vec{x}|\right)}{\frac{4}{3} \pi r_{0}^{3}}
\end{aligned}
$$

where we have neglected the suffix $\Sigma_{A_{0}}$. If we now change coordinate system and we move to the spherical coordinates $(r, \theta, \phi)$ where

$$
\vec{\nabla} f(r, \theta, \phi)=\frac{\partial f}{\partial r} \hat{e}_{r}+\frac{1}{r} \frac{\partial f}{\partial \theta} \hat{e}_{\theta}+\frac{1}{r \sin \theta} \frac{\partial f}{\partial \phi} \hat{e}_{\phi}
$$

we obtain the following result

$$
\begin{aligned}
\langle\tilde{S}\rangle_{A_{0}, r_{0}}-\langle S\rangle_{A_{0}, r_{0}}= & \frac{\int d \phi d \theta d r r^{2} \sin \theta \bar{S}^{(1)} \theta\left(r_{0}-r\right)}{\frac{4}{3} \pi r_{0}^{3}} \frac{\int d \phi d \theta d r r^{2} \sin \theta \frac{\partial}{\partial r} \epsilon_{(1)} \delta\left(r_{0}-r\right)}{\frac{4}{3} \pi r_{0}^{3}} \\
& -\frac{\int d \phi d \theta d r r^{2} \sin \theta \bar{S}^{(1)} \frac{\partial}{\partial r} \epsilon_{(1)} \delta\left(r_{0}-r\right)}{\frac{4}{3} \pi r_{0}^{3}}
\end{aligned}
$$

If $\bar{S}^{(1)} \neq 0$ and we take a radius of integration $r_{0}$ which is small compared with the typical size of our cosmological perturbations $r_{*} \sim 100 \mathrm{Mpc}$, the integral of such first order perturbation is non zero and, as said, the gauge dependence will be subleading with respect to this first order contribution.

Let us now consider a region of integration with $r_{0}>r_{*}$ and make the hypothesis that we can set to zero the integral of first order quantities. Then, following [16], we connect our fluctuations with Gaussian primordial ones with zero mean and we use ensemble average $([17,18])$ to calculate the expectation value of our averages. Following the standard notation we denote this additional average by an over-bar.

Let us consider the momentum expansion of a general first order perturbation $\sigma^{(1)}$ in the form

$$
\sigma^{(1)}(\vec{x})=\frac{1}{(2 \pi)^{3 / 2}} \int d^{3} k \mathrm{e}^{i \vec{k} \cdot \vec{x}} \sigma_{k}^{(1)} E(\vec{k})
$$


Using such expansion and the properties mentioned above in the Eq.(4.7) the first order integrals disappear and one obtains

$$
\begin{aligned}
\langle\tilde{S}\rangle_{A_{0}, r_{0}}-\langle S\rangle_{A_{0}, r_{0}}= & -\left(\frac{4}{3} \pi r_{0}^{3}\right)^{-1} \frac{1}{(2 \pi)^{3}} \int d \phi d \theta d r r^{2} \sin \theta\left[\int d^{3} k_{1} d^{3} k_{2} e^{i \vec{k}_{1} \cdot \vec{r}}\right. \\
& \left.\bar{S}_{k_{1}}^{(1)} E\left(\vec{k}_{1}\right) i k_{2} \cos \Omega e^{i \vec{k}_{2} \cdot \vec{r}} \epsilon_{(1) k_{2}} E\left(\vec{k}_{2}\right)\right] \delta\left(r_{0}-r\right)
\end{aligned}
$$

where $\Omega$ is the angle between $\vec{k}_{2}$ and $\vec{r}$. If our fluctuations are statistically homogeneous, then $E$ is a unit random variable satisfying $E^{*}(\vec{k})=E(-\vec{k})$ and

$$
\overline{E\left(\vec{k}_{1}\right) E\left(\vec{k}_{2}\right)}=\delta\left(\vec{k}_{1}+\vec{k}_{2}\right) \text {. }
$$

It follows that the ensemble average of Eq.(4.9) gives

$$
\begin{aligned}
\overline{\left(\langle\tilde{S}\rangle_{A_{0}}-\langle S\rangle_{A_{0}}\right)}= & -\left(\frac{4}{3} \pi r_{0}^{3}\right)^{-1} \frac{1}{(2 \pi)^{3}} \int d \phi d \theta d r r^{2} \sin \theta \quad\left[\int d^{3} k \frac{\bar{S}_{k}^{(1)}}{S^{(0)}} i k\right. \\
& \left.\cos \tilde{\Omega} \epsilon_{(1) k}\right] \delta\left(r_{0}-r\right),
\end{aligned}
$$

where $\tilde{\Omega}$ is the angular separation between the vector $\vec{k}$ and $\vec{r}$. By considering the spatial integral inside the momentum integral, it is easy to check, by symmetry arguments, that the spatial integral vanishes and the gauge dependence goes to zero as a consequence of the assumptions made. This is not surprising, the assumption that our average over a limited region may be replaced with an ensemble average is reasonable only if we perform the integration on a region which is much bigger than the scale of our inhomogeneities. In this case the gauge dependence should, indeed, vanish ${ }^{1}$.

\section{Conclusion}

This paper is focused on different issues associated with the evaluation of the backreaction effects of the classical (quantum) inhomogeneities, as the residual gauge dependence of the averaging prescription introduced in $[5,6]$ and the choice of the observers with respect to whom we evaluate the effects.

In Sect.3 we have described how to construct a scalar field $A(x)$, homogeneous in a given gauge, which defines the hypersurface on which we want to perform the average thus introducing the tools needed to have a correspondence between the standard average prescription [7] and the one introduced in $[5,6]$. Such a scalar will identify the observers

\footnotetext{
${ }^{1}$ Actually, if one applies an ensemble average directly to Eq.(4.7), using Eqs.(4.8) and (4.10) and without setting to zero the integral of first order quantities, the first term on the r.h.s. can give a non-vanishing contribution which is however negligible in the limit $r_{0} \gg r_{*}$.
} 
with respect to whom the average is performed. The physical properties of such observers are independent from the zero mode of the scalar field and from the possible choice of gauge. We have a gauge invariant way to select the observers. As a key example we gave the scalar and the velocity vector which identify the observers in geodesic motion with respect to the perturbed FLRW space-time.

The results obtained in Sect.3 can also be used for the evaluation of the quantum cosmological backreaction. In fact, the classical averages performed over all 3-dimensional space can be replaced by quantum expectation values using the correspondence illustrated in $[5]$.

In Sect.4 we have considered and formalized the residual gauge dependence associated with the averaging prescription (introduced in $[5,6]$ ) when the average is performed on a finite volume. Let us recall again the main results: first, the result (4.3) is independent from the particular window function used. Second, we confirm (as already stated in [5]) that the gauge dependence goes to zero for averages over all the 3 -dimensional space. Third, regardless of the averaged scalar $S$, the gauge dependence is always at least of the second order. And, in the end, if $\bar{S}^{(1)}=0$ the average is gauge invariant up to second order.

\section{Acknowledgements}

I wish to thank Maurizio Gasperini and Gabriele Veneziano for several discussions and comments on the manuscript, Adam Christopherson for comments on the manuscript and Julien Larena for discussions on the ensemble average. I was supported by the GIS "Physique des Deux Infinis" during the first stage of this work.

\section{References}

[1] G. F. R. Ellis, in "10th International Conference on General Relativity and Gravitation", ed. by B. Bertotti, De Felice and A. Pascolini (Reidel, Dordrecht, 1984), p. 215.

[2] T. Buchert, Gen. Rel. Grav. 40, 467 (2008).

[3] T. Buchert, Jap. Gen. Rel. Grav. 9, 306 (2000); T. Buchert and M. Carfora, Phys. Rev. Lett. 90, 031101 (2003); S. Rasanen, JCAP 02 (2004) 003; E. W. Kolb, S. Matarrese, A Notari and A. Riotto, Phys. Rev. D 71, 023524 (2005); E. W. Kolb, S. Matarrese and A. Riotto, New. J. Phys. 8 (2006) 322. 
[4] J. M Bardeen, Phys. Rev. D 22, 1882 (1980).

[5] M. Gasperini, G. Marozzi, and G. Veneziano, JCAP 03 (2009) 011.

[6] M. Gasperini, G. Marozzi, and G. Veneziano, JCAP 02 (2010) 009.

[7] T. Buchert, Gen. Rel. Grav. 32, 105 (2000); 33, 1381 (2001).

[8] O. Umeh, J. Larena, and C. Clarkson, arXiv:1011.3959 [astro-ph.CO].

[9] N. Li and D. J. Schwarz, Phys. Rev. D 76, 083011 (2007).

[10] J. Larena, Phys. Rev. D 79, 084006 (2009).

[11] I. A. Brown, J. Behrend and K. A. Malik, JCAP 11 (2009) 027.

[12] G. F. R. Ellis and M. Bruni, Phys. Rev. D 40, 1804 (1989).

[13] M. Bruni, S. Matarrese, S. Mollerach and S. Sonego, Class. Quant. Grav. 14 (1997) 2585 .

[14] K. A. Malik and D. R. Matravers, Class. Quant. Grav. 25 (2008) 193001; K. A. Malik and D. Wands, Phys. Rept. 475, 1 (2009).

[15] F. C. Mena, D. J. Mulryne and R. Tavakol, Class. Quant. Grav. 24 (2007) 2721.

[16] C. Clarkson, K. Ananda, and J. Larena, Phys. Rev. D 80, 083525 (2009).

[17] Durrer, R. The Cosmic Microwave Background, Cambridge University Press, 2008.

[18] Liddle, A. R., and Lyth, D. H., Cosmological Inflation and Large-Scale Structure, Cambridge University Press, 2000. 